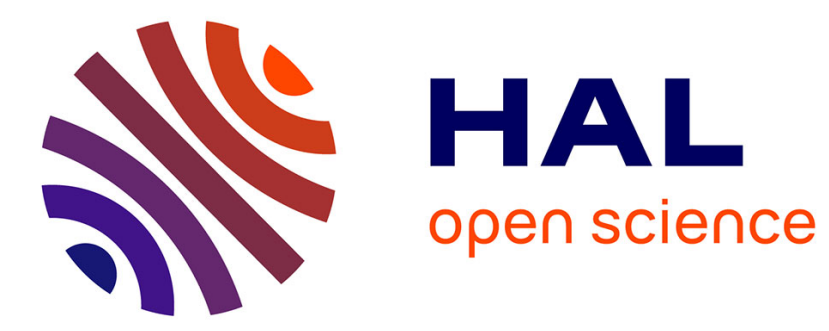

\title{
Bionanophotonics: general discussion
}

Amit Chandra, Amit Chattopadhyay, Richard Cogdell, Ankona Datta, Arijit De, Shaina Dhamija, Murali Golla, Stefan Haacke, Mahesh Hariharan, John Helliwell, et al.

\section{> To cite this version:}

Amit Chandra, Amit Chattopadhyay, Richard Cogdell, Ankona Datta, Arijit De, et al.. Bionanophotonics: general discussion. Faraday Discussions, 2018, 207, pp.491 - 512. 10.1039/C8FD90007H . cea-01894444

\section{HAL Id: cea-01894444 https://hal-cea.archives-ouvertes.fr/cea-01894444}

Submitted on 23 Oct 2019

HAL is a multi-disciplinary open access archive for the deposit and dissemination of scientific research documents, whether they are published or not. The documents may come from teaching and research institutions in France or abroad, or from public or private research centers.
L'archive ouverte pluridisciplinaire $\mathbf{H A L}$, est destinée au dépôt et à la diffusion de documents scientifiques de niveau recherche, publiés ou non, émanant des établissements d'enseignement et de recherche français ou étrangers, des laboratoires publics ou privés. 


\title{
Bionanophotonics: general discussion
}

\author{
Amitava Chandra, Amitabha Chattopadhyay, (D) Richard Cogdell, (D) \\ Ankona Datta, Arijit De, (D) Shaina Dhamija, Murali Golla, \\ Stefan Haacke, (D) Mahesh Hariharan, John Helliwell, (D) \\ Roberto Improta, Ramapurath S. Jayasree, Mike Jones, Joshy Joseph, \\ Tolga Karsili, Bern Kohler, Retheesh Krishnan, Imon Mandal, (DD \\ Dimitra Markovitsi, (D) Himani Medhi, Padmaja P. Mishra, Priyadarshi Roy \\ Chowdhury, Manas Sarangi, Ilme Schlichting, John Seddon, \\ Amit Sharma, Atchimnaidu Siriki, Rajaram Swaminathan, (D) Rienk van \\ Grondelle, Reji Varghese, Ravindra Venkatramani and Anthony Watts
}

DOI: 10.1039/C8FD90007H

Richard Cogdell opened a general discussion of the paper by Amitabha Chattopadhyay: The analysis depends on the spatial resolution, so when you classify them as dimers or trimers they have to be within a certain volume, so can you say that they are really dimers or are they just very close to each other?

Amitabha Chattopadhyay responded: The analysis depends on spatial correlation of the photobleaching data. The size of the oligomers is deduced from fitting the model.

Amit Sharma asked: What is the size of these clusters when they are dimers or trimers? Would it not be possible to obtain the size through FRET studies?

Amitabha Chattopadhyay answered: The size of the oligomers will be in the $\mathrm{nm}$ range. FRET will provide proximity (distance) information, not size.

Rajaram Swaminathan said: I have two questions. First, what role does lipid composition play in the results shown? Will results be different with another lipid composition? Second, are there reports in the literature on GPCR oligomerization in artificial liposomes where composition can be controlled?

Amitabha Chattopadhyay responded: To answer the first question, results from our laboratory and a number of groups have shown that lipid composition could play a role in GPCR function and oligomerization. To the second question, there are not too many examples of GPCRs oligomers studied in reconstituted liposomes. This is due to a variety of reasons which include difficulty in purification, and more importantly, lack of other cellular ingredients (necessary for oligomerization) in liposomal systems. 
Anthony Watts asked: Have you confirmed that EYGP does not influence the oligomerization state of the receptor? Have you any information on how oligomerization and cholesterol affects downstream signal processing, such as G protein coupling and bias coupling?

Amitabha Chattopadhyay replied: Yes - we have previously shown ${ }^{1}$ that attachment of EYFP does not alter the affinity and G-protein coupling of the serotonin-1A receptor. Moreover, the type of GFP molecules used by us do not show oligomerization under these conditions. We have previously published how cholesterol affects downstream signaling. ${ }^{2}$ We plan to monitor the effect of oligomerization on dowmstream signaling.

1 T. J. Pucadyil, S. Kalipatnapu, K. G. Harikumar, N. Rangaraj, S. S. Karnik and A. Chattopadhyay, Biochemistry, 2004, 43, 15852-15862.

2 T. J. Pucadyil and A. Chattopadhyay, Biochim. Biophys. Acta, 2007, 1768, 655-668.

John Helliwell communicated: Is there a GPCR serotonin crystal structure? If so is there a serotonin binding site between adjacent GPCRs, as well as to one GPCR? This would be an independent corroboration of the photobleaching image correlation spectroscopy method, which I assume is fairly new.

Amitabha Chattopadhyay communicated in response: The crystal structure of the serotonin-1A receptor is not available yet. However, crystal structures are available for a few other serotonin receptors. The crystal structure I showed in one of my slides where cholesterol molecules were shown to be bound between two monomers of a receptor was that of the $\beta 2$-adrenergic receptor.

Priyadarshi Roy Chowdhury communicated: How do you identify the cholesterol binding sites in the serotonin1A receptor?

Amitabha Chattopadhyay communicated in reply: By examining the presence of CRAC motifs in the sequence of the receptor. Please note that these are putative cholesterol binding sites.

Imon Mandal opened discussion of the paper by Ramapurath S. Jayasree: Does the NIR absorption depend on the length of the nanorod? If it does, how will that affect the photothermal activity of the GNR and the overall drug release?

Ramapurath S. Jayasree answered: Yes, the NIR absorption depends on the aspect ratio of the nanorod. In this case, the absorption maximum was around $786 \mathrm{~nm}$, which extends from 750 to $850 \mathrm{~nm}$. A $786 \mathrm{~nm}$ laser would have been ideal. However, our laser has a wavelength of $808 \mathrm{~nm}$ and hence we used this for photothermal activity.

Bern Kohler asked: Can you comment more on the chemistry that you use to attach the drug to the gold nanorod? Could you also elaborate on the mechanism of drug release? 
Ramapurath S. Jayasree replied: Thank you for the question. During the drug conjugation the formation of an ester bond is expected, which gets cleaved during drug release as the laser is applied. Drug release is hypothesized to occur by ester hydrolysis which is observed by the increase in fluorescence of the drug on laser irradiation.

Bern Kohler queried: The UV-visible absorption spectra in Fig. 1a in your paper appear to show a decrease in absorbance below $325 \mathrm{~nm}$ when the mitoxantrone drug is taken up by the gold nanorods, following treatment with folic acid. Both folic acid and mitoxantrone show strong absorption in the UV, so why doesn't the presence of both lead to the greatest absorption?

Ramapurath S. Jayasree answered: The reaction strategy adopted was such that conjugation of the drug was the final process in the system, which already has folic acid conjugated. The amount of drug used is half the concentration of folic acid so as to retain its identity. In the IR spectrum ester bond formation is visible in the final system; this ester bond may be formed between one of the carboxylic groups of folic acid and $-\mathrm{OH}$ of the mitoxantrone drug. This could be the reason for the decrease in absorption at $325 \mathrm{~nm}$. From the peak at $275 \mathrm{~nm}$, the presence of folic acid can be confirmed.

Anthony Watts asked: Are you sure that you have sufficient resolution to confirm co-localization of gold nanoparticles and nuclear marker, bearing in mind the Abbye diffraction limit problems when using co-localization of images?

Ramapurath S. Jayasree replied: Thank you for the question, we agree with your concern. The microscope had a resolution limit of $200 \mathrm{~nm}$, which is nowhere near the size of the nanorod. Moreover, diffraction limit issues are prominent at red emission and hence need special attention in order to capture the image. Please note that we don't claim to visualize the co-localization of gold nanorods by performing the nuclear stains. We haven't visualized the rods as such inside the cell. But, we have considered the red fluorescence signal originating from the gold nanorods for ascertaining the co-localization of the material inside the cell. This is very clear from the images, which were acquired by optimizing the parameters and applying aberration corrections.

Atchimnaidu Siriki said: Your final target molecule which you used for photothermal treatment shows high fluorescence, how will that molecule be useful for photothermal treatment? Did you calculate the quantum yield for that final molecule? And what concentrations of final molecule were used for photothermal treatment?

Ramapurath S. Jayasree answered: The final material MTX@FPGNR is used for photothermal treatment in the bound stage which has comparatively low fluorescence (the result shown in the fluorescence spectrum is normalised). We didn't calculate the quantum yield. We are planning to do it soon, before doing any in vivo experiments. On laser irradiation, the drug is released from the gold nanorod and the inherent free electron transition of the rod gets exposed, facilitating its 
photothermal properties. We used a $1 \mathrm{mg} \mathrm{ml}^{-1}$ solution of the final product for photothermal therapy.

Reji Varghese commented: The efficacies of PTT and chemotherapy have to be separately investigated. What is the advantage of combined therapy versus individual therapy?

Ramapurath S. Jayasree answered: Thank you for the question. The efficacies of PTT and chemotherapy have been investigated separately in c6 glial cells and HeLa cells and results are given in the ESI† of our article. Fig. S6 $\dagger^{\dagger}$ gives an idea regarding the efficacy of chemotherapy alone while Fig. S7† gives an idea of PTT alone (GNR - represented by blue bars). It is clear from these results that the individual therapeutic approaches have less efficiency compared to the combined approach. The intention of having a dual modal therapeutic approach was to increase the efficiency, which we could achieve. I hope this response is clear, if not I will be happy to provide more explanation.

Joshy Joseph asked: Can you comment on the extent of anti-cancer drug loading onto these functionalized gold nanorods and their release with/without the laser irradiation?

Ramapurath S. Jayasree replied: Thank you for the question. The loading capacity of the system is approximately $675 \mu \mathrm{M}$. Without laser irradiation there is no drug release (see Fig. S2 in the ESI $\dagger$ ). Drug release is qualitatively evaluated by measuring the increase in fluorescence of the drug (the cleavage of ester bonds formed during drug conjugation with GNR is predicted as we observe an increase in the fluorescence of the drug on laser irradiation, which initially got quenched on conjugation with the gold rods). Drug release with and without laser irradiation at two different $\mathrm{pH}$ values is given in Fig. $\mathrm{S} 4 . \dagger$

Himani Medhi communicated: This is a nice piece of work. This work will be helpful with further deep investigations if it can be applied directly to the target cancerous cells in a living system.

Ramapurath S. Jayasree communicated in reply: Thank you for the comment. We are working on it further to extend it to preclinical testing and if possible to clinical trials. Before which we have to collect the experimental data which are lacking, such as the quantum yield of the final system, stability, in vitro drug release profile, etc.

John Helliwell communicated: For the method described in Section 2.2.2 on page 4 of your article ("in vitro drug release study"), what percentage of the mitoxantrone is released from the gold nanorods on irradiation with the $808 \mathrm{~nm}$ laser for $1.3 \mathrm{~s}$ with $1 \mathrm{~W}$ power? This value would I presume be the best that can be achieved, and therefore it is important to know it, since in vivo I imagine the irradiation induced release of the drug will be less efficient as a process.

Ramapurath S. Jayasree communicated in response: In the case of in vitro drug release, the percentage drug release depends on the total amount of the final 
material used. When used in cells, more than $50 \%$ of (what we have used) drug release was observed. In the case of in vivo drug release, we agree with Prof. Helliwell that release will be less due to various reasons; but it can be controlled by suitably changing the total concentration of final material and the irradiation time. The concentration of material which would be used for in vivo studies will be optimized in future studies.

Joshy Joseph opened a general discussion of the paper by Ankona Datta: I have two questions. First, the authors have done theoretical calculations to find out the optimum distance (between amino acid residues) for placing the FRET pair for effective interactions. Do these calculations also account for the flexible spacer, which can change the effective distance between the FRET pair depending on different confirmations? Second, have the authors tried placing the fluorophore in the neighboring amino acids to check whether it is more/less effective than the computationally selected amino acid residues? This will probably ascertain the role of the flexible spacer.

Ankona Datta answered: To answer the first question: the donor tryptophan was a part of the peptide that we synthesized. The acceptor IAEDANS was conjugated to the peptide via a flexible linker. We have not included either the tryptophan or the flexible linker in our calculations which examine the original Gel 150-169 sequence. While the presence of the linker can change the effective distance between the FRET pair, we do find that the FRET efficiency increases as the peptide undergoes the coil-helix transition upon binding $\mathrm{PI}(4,5) \mathrm{P} 2$ as predicted by the distance changes captured in the simulations. For the second question: this is a nice suggestion; we plan to do these experiments in the future. However we would also like to mention that the computations suggested multiple residue pairs like residues 11-18 and residues 10-18 which showed maximal inter-residue distance changes during peptide coil-helix transitions. In the present study, we selected residues 11-18 for our experimental investigations.

Padmaja P. Mishra asked: What is the smallest number of amino acids where you see this structural change?

Ankona Datta answered: The structural change is observed in the Gel 150-169 peptide sequence which is a 20 amino acid peptide. In the future, we could study shorter fragments of the peptide to check if the peptide can retain its helical form upon binding to lipids.

Arijit De commented: Very nice work! I guess the positions (11 and 18?) of the FRET pairs are on the same side of the helix - is that right? If so, during the coil to helix transition, when the helix starts to unfold, the angle between the transition dipoles of FRET pairs should also change which, apart from a change in the point to point distance, should change the FRET efficiency.

Ankona Datta answered: Thank you for appreciating our efforts. The residues 11 and 18 on the 20 amino acid Gel 150-169 correspond to Glutamine 160 and Glycine 167. These residues are on the same side of the helix. In order to evaluate the effect of the orientation of these residues on the FRET efficiency we also 
calculated the relative orientation of Glutamine 160 and Glycine 167 for 5000 conformations of the helical and coil peptide states obtained from the MD simulations. The relative orientation of residues was defined as the angle between the vectors normal to the plane containing the $\mathrm{C} \alpha$ and the backbone carbonyl group of the residues. The average orientation of Glutamine 160 and Glycine 167 peaked at around $30^{\circ}$ for the helical conformers while the orientations for the coil conformers were random. This result has been summarized in our manuscript in Fig. 3 and indicates that the relative orientation of the two residues would favor increased FRET efficiency in the helical conformers. However, since we attached the acceptor via a flexible linker which is not present in the simulations, the effect of the favorable orientation cannot be directly assessed.

Anthony Watts asked: How sure are you that the peptide-membrane association is not a simple electrostatic association, and have you examined other anionic lipids? Your salt concentrations and ionic strengths are sufficiently high in the experiment that all charges are shielded.

Ankona Datta responded: We have examined the binding of the Gel 150-169 peptide with different anionic phospholipids in our previous work. ${ }^{1}$ The binding affinity of the peptide is highest for $\mathrm{PI}(4,5) \mathrm{P} 2\left(K_{\mathrm{d}} 3 \mu \mathrm{M}\right)$. Other anionic phospholipids like phosphatidylserine $\left(K_{\mathrm{d}}>100 \mu \mathrm{M}\right)$ and phosphatidylglycerol have much lower binding affinities to the peptide. We have performed all experiments at physiological pH 7.4 using $20 \mathrm{mM}$ HEPES buffer and $100 \mathrm{mM} \mathrm{NaCl}$. These results indicate that the peptide-membrane interaction is not a simple electrostatic association. We believe that the positively charged residues in the Cterminus of the peptide are sequentially arranged for optimal electrostatic interaction with PI(4,5)P2. However, the interactions of the hydrophobic residues near the N-terminus are also important for the selectivity. When we delete residues from the N-terminus we observe that the selectivity of the peptide toward PI(4,5)P2 is slightly lowered. ${ }^{1}$ For example, the 13 amino acid peptide Gel 157-169 affords a $K_{\mathrm{d}}$ value of $1.4 \mu \mathrm{M}$ with $\mathrm{PI}(4,5) \mathrm{P} 2$ and $14 \mu \mathrm{M}$ with PS. We have also performed molecular dynamics simulations on the helical conformer of the Gel 150-169 peptide in the presence of a lipid bilayer containing $0.74 \mathrm{~mol} \%$ of $\mathrm{PI}(4,5)$ $\mathrm{P} 2$ on one leaflet using explicit solvent and $120 \mathrm{mM} \mathrm{NaCl}$ to mimic our experimental conditions. The simulation results indicated that the arginine residues at the peptide C-terminus formed stable salt bridge interactions with the PI(4,5)P2 headgroups throughout the entire timescale of the simulation at these ionic strength conditions, further corroborating our experimental observations.

1 S. Mondal, A. Rakshit, S. Pal and A. Datta, ACS Chem. Biol., 2016, 11, 1834-1843.

Anthony Watts asked: A unique PI(4,5)P2 binding site in your peptide would be a unique finding - is it found elsewhere?

Ankona Datta answered: The Gel 150-169 sequence is a PI(4,5)P2 binding site for the actin binding protein Gelsolin. This had been shown by Paul Janmey and his co-workers earlier. ${ }^{1}$ Our studies on the Gel 150-169 peptide tagged to a polarity sensitive dye allowed the determination of $K_{\mathrm{d}}$ values for the interaction of the peptide with lipid vesicles containing different negatively charged 
phospholipids. $^{2}$ The results indicated highest affinity of the peptide toward PI(4,5)P2 followed by PI4P. Other negatively charged phospholipids like phosphatidylserine showed 30 times lower affinity to the peptide. Actin binding proteins like Villin and Profilin also have sequences that are similar to Gel 150169 which are proposed to be phosphoinositide binding sites. ${ }^{3}$ Whether these sites are selective toward $\mathrm{PI}(4,5) \mathrm{P} 2$ can be investigated in the future.

1 W. Xian, R. Vegners, P. A. Janmey and W. H. Braunlin, Biophys. J., 1995, 69, 2695-2702.

2 S. Mondal, A. Rakshit, S. Pal and A. Datta, ACS Chem. Biol., 2016, 11, 1834-1843.

3 P. A. Janmey, W. Xian and L. A. Flanagan, Chem. Phys. Lipids, 1999, 101, 93-107.

Amitabha Chattopadhyay commented: You could change the ionic strength. This would help in ruling out electrostatic factors.

Ankona Datta responded: The interaction of the Gel 150-169 peptide with phosphatidylinositol $(4,5)$ bisphosphate $(\mathrm{PI}(4,5) \mathrm{P} 2)$ is primarily based on electrostatic interactions between lysine and arginine residues near the C-terminus of the peptide along with hydrophobic interactions with residues close to the $\mathrm{N}$ terminus of the peptide. ${ }^{1}$ In the present manuscript, we have also performed molecular dynamics simulations on the helical conformer of the Gel 150-169 peptide in the presence of a lipid bilayer containing $0.74 \mathrm{~mol} \%$ of $\mathrm{PI}(4,5) \mathrm{P} 2$ on one leaflet using explicit solvent and $120 \mathrm{mM} \mathrm{NaCl}$ to mimic the experimental conditions which utilize physiological ionic strengths. The simulation results indicated that the arginine residues formed stable salt bridge interactions with the PI(4,5)P2 headgroups throughout the entire timescale of the simulation. Previous work from our group ${ }^{2}$ indicates that the binding affinity of the peptide is highest for PI(4,5)P2 $\left(K_{\mathrm{d}} 3 \mu \mathrm{M}\right)$. Other anionic phospholipids like phosphatidylserine $\left(K_{\mathrm{d}}>100 \mu \mathrm{M}\right)$, phosphatidylglycerol, and phosphatidylethanolamine have much lower binding affinities to the peptide. We believe that the positively charged residues in the C-terminus of the peptide are sequentially arranged for optimal interaction with $\mathrm{PI}(4,5) \mathrm{P} 2$. Further, the interactions of the hydrophobic residues near the $\mathrm{N}$-terminus are also important for the selectivity. When we delete residues from the N-terminus we observe that the selectivity of the peptide toward PI(4,5)P2 is slightly lowered. For example the 13 amino acid peptide Gel 157-169 affords a $K_{\mathrm{d}}$ value of $1.4 \mu \mathrm{M}$ with $\mathrm{PI}(4,5) \mathrm{P} 2$ and $14 \mu \mathrm{M}$ with PS. ${ }^{2}$ As suggested by Prof. Chattopadhyay it would be interesting to perform experiments to study the effect of ionic strength on the salt-bridge interactions between the $\mathrm{PI}(4,5) \mathrm{P} 2$ headgroup and the peptide.

1 I. Liepina, C. Czaplewski, P. Janmey and A. Liwo, Biopolymers, 2003, 71, 49-70. 2 S. Mondal, A. Rakshit, S. Pal and A. Datta, ACS Chem. Biol., 2016, 11, 1834-1843.

Ilme Schlichting remarked: Your explanation of specificity relies on hydrophobic interactions of the $\mathrm{N}$ - of the peptide with the membrane and H-bonds/ saltbridges of the C-terminus of the peptide with PI(4,5)P2. In holo-gelsolin these interactions are not possible since the peptide is part of the protein. Can you comment, please?

Ankona Datta responded: The interaction of the Gel 150-169 peptide with $(\mathrm{PI}(4,5) \mathrm{P} 2)$ is primarily based on electrostatic interactions between lysine and 
arginine residues near the C-terminus of the peptide along with interactions with hydrophobic residues close to the N-terminus of the peptide. The peptide used for all experiments and also for a sub-set of the molecular dynamics simulations had an amide moiety in the C-terminus. This would also be the situation when the peptide is embedded in the protein. Importantly, the stable salt-bridge interactions of the residues in the C-terminus with $\mathrm{PI}(4,5) \mathrm{P} 2$ were with arginine side chains which would also be present in the protein. The C-terminus amide did not show any interactions with the $\mathrm{PI}(4,5) \mathrm{P} 2$ headgroup. In the same vein, the hydrophobic residues toward the N-terminus of the peptide came closer to the membrane as a result of the binding of the C-terminus with the PI(4,5)P2 headgroup. Both the electrostatic and hydrophobic interactions were with the side chains of amino acid residues. These interactions could also occur in the holoprotein.

Tolga Karsili said: Constant temperature and pressure dynamics are known to under-sample low-energy conformations of proteins. Are you confident that the dominant conformations are sufficiently sampled in the propagation time window reported in your manuscript?

Ankona Datta answered: In our peptide simulations, we employ Langevin temperature control and Nosé Hoover Langevin piston pressure control which rigorously produce canonical sampling. Nevertheless, the conformation space for peptides is vast and rigorous sampling of all conformational states may require simulation trajectories spanning hundreds of microseconds. ${ }^{1}$ However, the objectives of the peptide simulations in the present manuscript are far simpler: to sample inter-residue separations for two specific conformations (helix and coil forms) of the peptide. Circular dichroism experiments indicate that the Gel 150169 peptide and similar peptides from phospholipid binding sites of actin binding proteins adopt a coil conformation in buffer in the absence of lipids. ${ }^{2}$ In contrast, NMR experiments in organic solvents captured a helical conformation for Gel 150-169 which is the relevant lipid binding form for the peptide. ${ }^{2} \mathrm{We}$, therefore directly sampled the helix form at $300 \mathrm{~K}$ and used high temperature (500 K) simulations to unfold the peptide and generate the extended coil form (Fig. 1 in the present manuscript). Subsequently we used 5000 conformations over $10 \mathrm{~ns}$ from both the coil and helix states to calculate and contrast average inter-residue distances (Fig. 2 in the present manuscript). The average distances obtained from simulations provided appropriate guidelines for the design of a peptide based fluorescence resonance energy transfer sensor. The experimental data from steady state and time-resolved fluorescence proved that the guidelines obtained from the computations were optimum. Hence, we can confirm that we have sufficiently sampled inter-residue distances in the two relevant conformations of the Gel 150-169 peptide in our simulations.

1 Y.-S. Lin and V. S. Pande, Biophys. J., 2012, 102, 315-324.

2 W. Xian, R. Vegners, P. A. Janmey and W. H. Braunlin, Biophys. J., 1995, 69, 2695-2702.

John Seddon opened a general discussion of the papers by Amitabha Chattopadhyay, Ramapurath S. Jayasree and Ankona Datta: Can you identify the common themes of the session? 
Amitabha Chattopadhyay replied: Application of bionanophotonics to biomedical problems.

Ramapurath S. Jayasree added: Bionanophotonics is an emerging field in biomedical science.

Ankona Datta responded: Using biophysical and chemical tools to elucidate molecular mechanisms underlying biological processes and further using the insights obtained to develop tools for theranostic applications.

Amitava Chandra addressed Ramapurath S. Jayasree: You mentioned that the anticancer agents are incorporated into the cell by the folic acid pathway, could you talk more about this pathway? Is there any specific interaction that is related to the incorporation of the materials into the cell?

Ramapurath S. Jayasree replied: Thank you for the question. The concept of folate receptor mediated delivery of drugs and drug carrying systems is well established. There are several articles in this line.

Atchimnaidu Siriki said: Related to the drug release at pH 7 and pH 4 after treatment with an $808 \mathrm{~nm}$ laser, you have shown that release of the drug at pH 4 is very high, if you consider a cancer cell will it have that $\mathrm{pH} 4$ in every organelle? And which part of the cancer cell is your final molecule entering?

Ramapurath S. Jayasree answered: It is well known that the extracellular region itself shows acidic $\mathrm{pH}$ in tumors. ${ }^{1}$ When the cell organelles are considered, the lysosomal bodies of cells are more acidic and $\mathrm{pH}$ will be less than 4 . In the cell uptake studies where we used the nuclear stains, we found that the drug enters more into the cytoplasm, though we expect entry into the nucleus as the drug used is expected to intercalate with DNA. A detailed time bound study in this direction is required to know more about this.

1 Y. Kato, S. Ozawa, C. Miyamoto, Y. Maehata, A. Suzuki, T. Maeda and Y. Baba, Cancer Cell Int., 2013, 13, 89.

Arijit De said: When you shine laser on the gold nanorod-drug (doxorubicin) construct, which is already inside the cancer cell, the cell can die either because of release of the drug or because of heating alone. My question is: can you pinpoint what is actually killing the cell, the free drug or the laser-induced heating? Now, as you said, there is a specific pH-dependent chemistry that leads to the cleavage of the ester bond releasing the drug; one can still wonder why that particular bond cleaves under irradiation, from the reaction dynamics point of view of unimolecular dissociation, but that is a fairly complicated process to have a simple answer.

Ramapurath S. Jayasree answered: Thank you for the good question. The whole purpose of using multimodal imaging and therapy is to increase the efficiency of both. In this study there are two simultaneous therapeutic approaches as well as two imaging approaches utilizing the same system. As you can see from Fig. S5 
and S6 of the ESI $\dagger$ of our article the therapeutic effects of drug alone and PTT alone are much less than the combined effect, which fulfills the intended purpose of increasing the therapeutic effect. So, the cell death happens due to the effect of both drug release and laser induced heating.

Talking about the chemistry, the formation of the ester bond on drug conjugation is clear and is observed in the IR spectrum as well. The cleavage is predicted as we observe an increase in the fluorescence of the drug on laser irradiation, which initially got quenched on conjugation with the gold rods. Please note that MTX is the only molecule which has sufficient NIR fluorescence in the whole system. So we assume that the drug regains its identity on laser irradiation by ester hydrolysis, increasing the fluorescence. The acidic $\mathrm{pH}$ of the cells favors the reaction. Due to laser irradiation there may be dissociation of other bonds also. However, no major changes were observed and also a detailed study of this aspect is not considered in this study.

Mahesh Hariharan addressed Ankona Datta: I have a general question related to the FRET pair that is often used for probing biological environments. Do you also consider aggregation of peptides, where you can have energy transfer from one peptide to another peptide that might give wrong information?

Ankona Datta answered: It is true that aggregation of peptides can lead to intra-molecular FRET. In order to avoid aggregation affects we have performed all of our experiments at low $\mu \mathrm{M}$ concentrations of the peptide.

Mahesh Hariharan asked: How much influence will the acceptor/donor chromophore have in steady state emission when surrounded by the lipids?

Ankona Datta replied: In our studies we observe that the acceptor IAEDANS exhibits polarity sensitive emission. IAEDANS is placed close to the C-terminus which directly attaches to the $\mathrm{PI}(4,5) \mathrm{P} 2$ headgroup through strong electrostatic interactions. Hence the steady state emission of IAEDANS is affected by the presence of lipids. However the donor tryptophan emission is not affected by the presence of lipids. The tryptophan residue is placed further away from the Cterminus towards the center of the peptide and both steady-state fluorescence and lifetime measurements indicate that the tryptophan emission is unaffected by the presence of lipids. Hence our conclusions on peptide conformational changes upon lipid binding have been drawn on the basis of the tryptophan emission data.

Rajaram Swaminathan said: I am interested in what is happening inside the living cell compared to in vitro measurements. Could you comment on the possibility of performing FRET measurements inside the cell?

Ankona Datta answered: In order to perform FRET experiments in living cells we would require FRET donor-acceptor pairs which absorb and emit in the visible wavelengths. We plan to explore such FRET pairs in the future.

Ravindra Venkatramani addressed Amitabha Chattopadhyay: Extrapolating the results in your paper, is it possible to systematically vary cholesterol 
concentration to selectively obtain monomer, dimer, trimer, and higher order oligomer populations for the GPCRs in membranes?

Amitabha Chattopadhyay responded: This is more involved and complicated. Different cell types have varying cholesterol content. Moreover, upon cholesterol replenishment, one is not sure whether cholesterol is put back from where it was depleted. These factors make the situation more complicated. A simple extrapolation is not possible.

Retheesh Krishnan addressed Ramapurath S. Jayasree: In your paper you have mentioned that the weak emission of PGNR and FPGNR are enhanced in the presence of PEG and folic acid. You have also mentioned that two strong emission peaks are present for the MTX@FPGNR. As the spectrum is normalized the strength of emission is not visible in it. You are using Raman to detect the delivery of the drug. Doesn't the strong emission of MTX@FPGNR affect your Raman measurements?

Ramapurath S. Jayasree answered: Thank you for the question. The data prior to normalization is shown here in Fig. 1. Please note that the fluorescence of the gold nanorods increases in PGNR and FPGNR. This increase is nominal with respect to fluorescence intensity. On addition of the drug mitoxantrone the fluorescence further increases. This could be due to energy transfer from gold to the drug (this we have not considered in detail and it is not explained in the paper). However, this increased fluorescence itself was not very strong as you see from the $y$-axis. Hence we could acquire Raman spectra without hindrance from

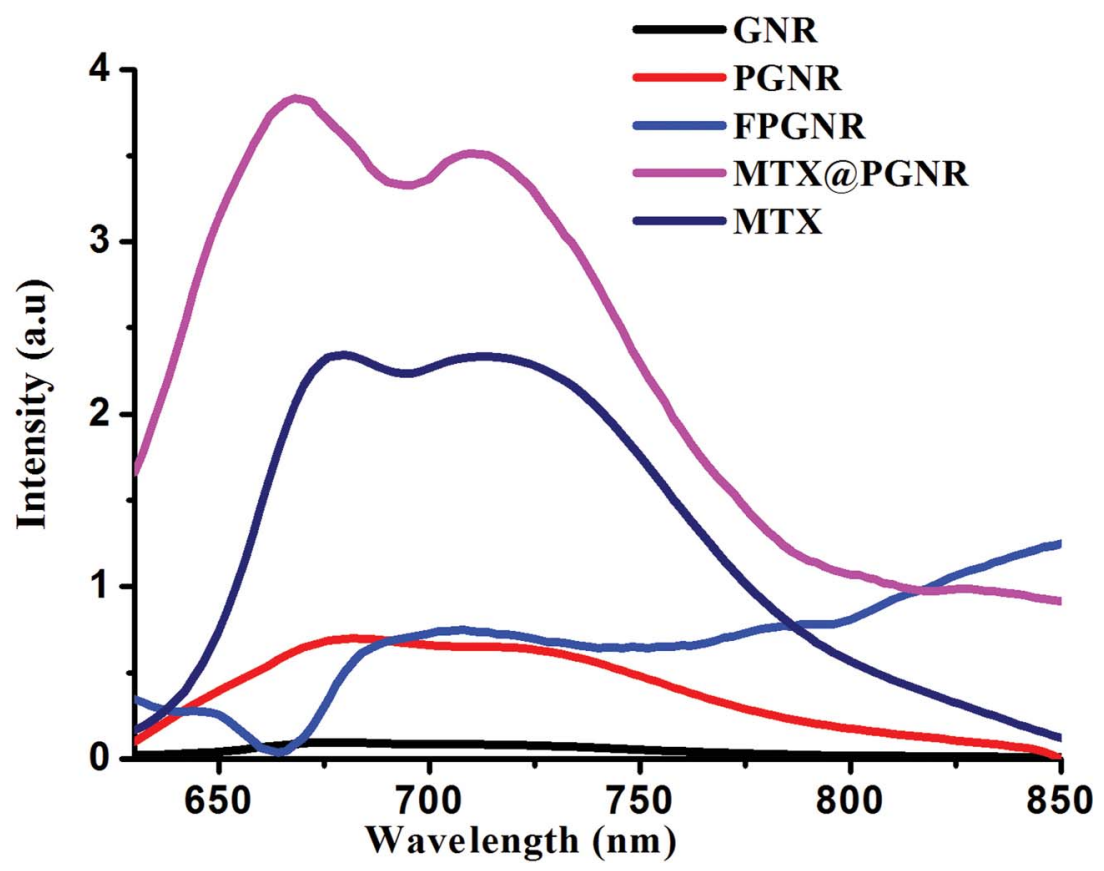

Fig. 1 Emission spectra of GNR, PGNR, FPGNR and MTX@FPGNR before normalization. 
the fluorescence, initially. Moreover, the presence of GNR helps to enhance the Raman signal by many fold through the mechanism of SERS.

Imon Mandal opened a general discussion of the paper by Joshy Joseph: What is the molecular basis of the interaction (e.g. electrostatic, H-bonding etc.) between F-An and DNA molecules? How will the DNA absorption change if the $\mathrm{pH}$ of the F-An solution is altered?

Joshy Joseph replied: The functionalised fullerene (F-An) derivative containing aniline and fullerene subunits self-assembles in 10\% DMSO-PBS mixture into 3$5 \mathrm{~nm}$ sized nanoclusters with the aniline groups projecting outside. Aniline remains neutral at physiological $\mathrm{pH}$ conditions and can bind to the grooves (preferably the minor groove) of DNA through H-bonding and van der Waals interactions. The initial interaction of F-An clusters with DNA is governed by the groove interactions of the pendent aniline moiety which might be sufficient enough to align the clusters along the grooves, facilitating further interaction of fullerenes through groove binding and $\pi-\pi$ interactions.

We have carried out all the experiments reported in this paper in $10 \mathrm{mM}$ phosphate buffer containing $2 \mathrm{mM} \mathrm{NaCl}$, at $\mathrm{pH}$ 7.4. At higher $\mathrm{pH}$ and lower $\mathrm{pH}$ conditions the B-form DNA structure may get perturbed. At lower $\mathrm{pH}(\mathrm{pH}<5)$ the aniline moieties in F-An can get protonated and an additional, electrostatic mode of binding can be expected.

Bern Kohler said: I have two questions. First, how monodisperse are the fullerene clusters and what prevents their further aggregation? Second, you refer to the decrease in absorbance seen when the fullerene nanoclusters are disassembled as a hypochromic effect, but in DNA disassembly of stacked nucleobases leads to an absorbance increase. How does interaction with the CT-DNA modify the $336 \mathrm{~nm}$ absorption band of the fullerene nanoclusters?

Joshy Joseph answered: Regarding the first question, the TEM studies of F-An in $10 \%$ DMSO-PBS mixture showed the formation of almost mono-disperse nanoclusters with size ranging from 3-5 $\mathrm{nm}$. Under similar conditions, other fullerene derivatives (F-Py and F-PTz) exhibited different self-assembly behavior (molecularly dissolved and larger nanoclusters, respectively). Hence, the amphiphilic structure and unique hydrophobic-hydrophilic balance of fullerene derivatives is responsible for their uniform clustered aggregation behavior. The clusters may be considered as a fullerene core on the inside and aniline appended triethyleneglycol group projecting outside. Therefore, further aggregation will be prevented due to the presence of the more hydrophilic periphery of the clusters.

To answer the second question, the sequential addition of CT-DNA to F-An solution showed a regular increase in the absorption at $260 \mathrm{~nm}$ corresponding to the absorption of the DNA bases with a concomitant decrease in the F-An nanoclusters absorption at $336 \mathrm{~nm}$. The observed changes (hypochromism at $336 \mathrm{~nm}$ ) could be due to the binding of F-An nanoclusters with CT-DNA through groove binding interactions of fullerene and aniline moieties.

The disassembly of CT-DNA/fullerene nanoclusters was not presented in this report. However, according to our previous report, ${ }^{1}$ we observed that disassembly of F-An nanoclusters from DNA during DNA melting studies led to an increase in 
the absorption at $260 \mathrm{~nm}$ with concomitant enhancement in the tail-end absorption (400-600 $\mathrm{nm})$.

1 S. K. Vittala, S. K. Saraswathi and J. Joseph, Chem.-Eur. J., 2017, 23, 15759-15765.

Reji Varghese asked: Is the space available in the groove (minor or major) sufficient enough for a cluster with a size of 3-4 $\mathrm{nm}$ to bind effectively?

Joshy Joseph answered: In this paper, we have used functionalised fullerene (FAn) containing aniline and fullerene sub-units which in the presence of $10 \%$ DMSO-PBS mixture preferably self-assemble into 3-5 $\mathrm{nm}$ sized nanoclusters with aniline groups projecting outside. As we mentioned in the paper, both aniline and fullerene are known to interact with DNA through groove interactions. In the case of fullerene clusters, based on the observed absorption changes and the induced circular dichroism (ICD) for CT-DNA/F-An complexes corresponding to the F-An absorption (336 nm), we proposed a possible groove binding of F-An clusters along the CT-DNA templates. The fullerene clusters $(3-5 \mathrm{~nm})$ as such may be slightly large to fit on the DNA grooves. However, the initial interaction of F-An clusters with DNA is governed by the groove interactions of the pendent aniline moiety which might be sufficient enough to align the clusters along the grooves facilitating further interaction of fullerenes through groove binding and $\pi-\pi$ interactions.

Padmaja P. Mishra said: There are many experimental/computational efforts in this area where people have found unwinding or unzipping of DNA, while interacting with small charged molecules/particles. Do you find any such effect here? Does it bind to single stranded DNA?

Joshy Joseph responded: As reported previously, the unzipping or unwinding of DNA with fullerene can happen in the presence of external stimuli such as temperature or light. In the case of CT-DNA/F-An nanoclusters, morphological analysis revealed a perturbation in the highly networked structure of CT-DNA to form a nanofibrous structure. This is mainly due to the reduction in the DNADNA interactions subsequent to the groove binding of the F-An nanoclusters.

The UV-visible interaction studies of F-An nanoclusters with short single stranded DNA showed no change in the fullerene absorption indicating the absence of any kind of interactions with single strand DNA.

Murali Golla said: I have two questions. First, the CD signal of any DNA will depend on the conformation of the DNA. Generally CT-DNA show a bisignate CD signal, in your article after decoration of fullerene nanoclusters on CT-DNA, a positive CD signal was observed. Please could you explain what is the reason for there being only a positive CD signal? Second, after incubation of fullerene nanoclusters with CT-DNA, the entangled network was changed to a small network kind of morphology. Can you justify what is the reason for the change in morphology?

Joshy Joseph responded: In the present case upon increasing the concentration of F-An nanoclusters from $1: 0$ to $1: 1$, the CD spectra exhibited strong 
induced $\mathrm{CD}$ around $250-370 \mathrm{~nm}$, which is the region corresponding to F-An nanocluster absorption. Since, F-An absorption is in line with that of DNA, the observed positive induced CD signal could be attributed to the chiral organization of the F-An nanoclusters on the CT-DNA template, facilitated by the groove binding interactions and possible $\pi-\pi$ interactions between adjacently bound FAn nanoclusters.

To answer the second question, the CT-DNA/F-An complex exhibited a significantly different nanofibrous structure, where the highly networked structure of CT-DNA was broken down into smaller nanofibers with sizes of 50-100 $\mathrm{nm}$. The change in morphology of CT-DNA upon F-An nanocluster binding could be attributed to the reduction in the DNA-DNA interactions subsequent to the groove binding of the F-An nanoclusters.

John Helliwell communicated: You state in your abstract that 10\% DMSO was needed to dissolve the fullerene. What is the impact of $10 \%$ DMSO on the DNA (in the absence of fullerene)? Also, is "PBS" phosphate based saline?

Joshy Joseph communicated in reply: As stated in our manuscript, 10\% DMSO-PBS solvent mixture was used in all experimental studies. Before carrying out DNA interaction studies, the stability of CT-DNA in the presence of $10 \%$ DMSO-PBS and PBS alone were compared using circular dichroism (CD) studies. The unchanged CD signal in the presence of $10 \%$ DMSO-PBS refers to the intact B-form of DNA and hence this solvent mixture was used for further DNA interaction studies with fullerene derivatives. "PBS" refers to phosphate buffered saline. In the present case we have used $10 \mathrm{mM}$ phosphate buffer containing $2 \mathrm{mM} \mathrm{NaCl}$ at $\mathrm{pH}=7.40$.

Priyadarshi Roy Chowdhury communicated: What do you think about challenges in CT-DNA template analysis? What are the bottlenecks and how could these be overcome?

Joshy Joseph communicated in reply: The main challenge in the CT-DNA template analysis is the lack of information about nucleobase sequence structure and hence it is difficult to understand the specificity of binding. In addition, CT-DNA contains irregular lengths of nucleotide strands which cannot be used for the thermal denaturation studies. One can overcome these limitations by using specific oligonucleotide strands of known nucleotide sequences, in which case the exact nature of binding and accurate thermal denaturation can be studied.

Arijit De opened a general discussion of the paper by Richard Cogdell: Considering the molecular level understanding of energy transfer under low light (LL) conditions, a dark adapted strain shows shifting of energy levels in LH2 arresting the back-transfer of energy from LH1 (which is somewhat analogous to switching from carotenoid to chlorophyll energy transfer under LL conditions to chlorophyll to carotenoid energy transfer under high light (HL) conditions mediated via the xanthophyll cycle). On the other hand, the color change in LH2 is due to carotenoid incorporation. My question is: is there any specific role of these carotenoids or is the entire role still played by the chlorophylls alone, by tuning the coupling among themselves and with the protein, thereby shifting the absorption? 


\section{Discussions}

Apart from this "slow" change upon LL conditions, LH2 can also undergo "fast" conformation change (state transition) under strong pulse irradiation leading to dissipation of excess energy as heat, as revealed from the single complex studies by William Moerner and co-workers. ${ }^{1}$ In this case, the LH2 complex remains the same (there is no carotenoid incorporation). So, conformation change may be a more desired pathway for quickly adapting to changing light intensities. Can you please comment on that?

1. G. S. Schlau-Cohen, Q. Wang, J. Southall, R. J. Cogdell, W. E. Moerner, Proc. Nat. Acad. Sci. U. S. A., 2013, 110, 10899-10903.

Richard Cogdell replied: The effect of the carotenoid on the absorption of the $\mathrm{Bchl}$ a molecules is rather small. But often with LH2 complexes they are not stably assembled in the absence of carotenoids. And with all proteins LH2 does show conformational flexibility and this can be best seen in single molecule experiments. There is no evidence in the purple photosynthetic bacteria that any such conformational changes are used to modulate the efficiency of light harvesting. I should remind you that these are anaerobic photosynthetic organisms and unlike plants do not have a problem with too much light as no oxygen is present.

Amitava Chandra said: I'm interested in knowing the molecular picture of LH1 and LH2 complexes. What molecules are they and what specific molecular changes are happening?

Richard Cogdell responded: There are X-ray crystal structures for these pigment protein complexes. References to these are provided in our paper.

Anthony Watts remarked: Why does the bacterium not simply change the density of LHC membrane to capture more or less light, rather than have such a complex adaptation in various components, their concentrations, and their composition resulting in different kinetics and efficiency of light capture?

Richard Cogdell responded: They do of course make more membrane and more photosynthetic units as the light intensity in which they are grown is reduced. However, the more subtle regulation where they change the spectroscopic form of $\mathrm{LH} 2$ that is made is because the changes in the spectroscopic type of LH2 allows the excited state to reside longer on LH1 at low light and it to escape and find another LH1 complex at high light. This provides more efficient use of the absorbed photons under both conditions.

Rajaram Swaminathan asked: I am curious about the shift in the absorption spectrum towards the red wavelengths. Can it be shifted to the blue side too?

Richard Cogdell answered: In the example shown in this paper the B850 band in the high light form of LH2 is actually blue shifted to $820 \mathrm{~nm}$ in the low light growth conditions.

Rienk van Grondelle added: Following on from Richard Cogdell's explanation for why there is a blue shift. There is also a degeneracy factor, so the possibility to 
be in the central state is already small which in fact rate limits the trapping process. Doubling the antenna size would reduce this even further and for that reason the added antenna is maybe blue shifted.

Shaina Dhamija asked: Is it possible to achieve $\mathrm{pH}$-dependent spectral shift for LH2 (shifting the B850 band down to B830) instead of altering the light intensity?

Richard Cogdell answered: Most species of purple photosynthetic bacteria do not have LH2 complexes where the position of the B850 absorption band is $\mathrm{pH}$ sensitive in this way.

John Helliwell communicated: Can you please quantify the statement (on page 1 of your paper) that "the amount of ICM is a lot less"? On page 2, please can you quantify the statement "just a bit slower"?

Richard Cogdell communicated in reply: This is a very hard question to answer quantitatively. We are now doing some tomography to try to be more quantitative. The EM pictures only give a partial answer since the membranes appear not to be uniformly distributed throughout the cell. As to how much the LH2 to LH1 energy transfer step slows down on going from the HL to the LL LH2 complex, the rate slows from about 6 ps in the HL case to about 9 ps in the LL case. However since the Bchl a excited singlet state lifetime is about $1 \mathrm{~ns}$ these small changes have a very marginal effect on the overall energy transfer efficiency.

John Helliwell communicated: The method of varying the light intensity (described on page 4) is fine in the laboratory but in the natural environment absorbers of light such as water change the light spectrum as well as its intensity (a function of water depth and water type). Which environmental situation is the page 4 lab situation relevant to?

Richard Cogdell communicated in reply: The natural situation is so complicated because the light intensity varies, the spectral quality varies and of course there is the diurnal variation too. The lab situation is of course not the natural one, but it gives us the chance to dissect each effect individually. It is completely different to try to mimic the real natural system and far too complicated for me!

Priyadarshi Roy Chowdhury communicated: How do femtosecond dynamics affect the isolated LH2 complexes?

Richard Cogdell communicated in reply: The type of LH2 complexes we use are those that are the most stable. This means that they are not adversely affected by our femtosecond laser flash photolysis experiments.

Richard Cogdell addressed Joshy Joseph: One thing that is great about DNA is that you're not constrained by having single strand or double strand, you can produce origami structures. Have you thought about using DNA origami to produce ordered structures and place your fullerene derivatives on those? You could try to get well controlled patterns. 
Joshy Joseph answered: Thanks for the excellent suggestion. We also have thought about the possibility of using fullerene nanoclusters for organizing higher order DNA structures. In future studies we will try to investigate these aspects.

Joshy Joseph asked Richard Cogdell: Do the light absorbing chromophores have any change in their chemical structure in the cells grown under low light and high light conditions? How about the sizes of the LH1 and LH2 complexes grown under LL and HL conditions?

Richard Cogdell replied: The bacteriochlorophyll molecules remain the same. Sometimes as I have shown here in this paper the type of carotenoid can change. I do not know of an example of where the ring sizes change dependant on the growth conditions.

Rienk van Grondelle addressed Joshy Joseph: I have a question about fullerene. Fullerene clusters in heterojunction solar cells are used to remove electrons and the size of the cluster is an important factor in the cell's efficiency. Is there a role for your fullerene clusters on DNA (e.g. electronic) in terms of electron transfer properties?

Joshy Joseph answered: Yes. Fullerene derivatives were extensively used in bulk heterojunction solar cells as acceptors. Also, it is reported that aggregation of fullerenes in the active layer of the device will deteriorate the device performance. In our previous report, ${ }^{1}$ we observed that the nanowires obtained from the mutual assisted self-assembly of short DNA strands and fullerene clusters were semiconducting in nature. Here, the conductivity is mainly arising due to the presence of fullerene clusters and hence confirms the positive role of fullerene clusters in the electron transfer properties in DNA.

1 S. K. Vittala, S. K. Saraswathi and J. Joseph, Chem.-Eur. J., 2017, 23, 15759-15765.

Mike Jones asked: You mentioned in your paper there are a variety of ways of condensing DNA. Are there advantages to using a fullerene as compared to other methods of packaging?

Joshy Joseph replied: Fullerene derivatives have been extensively studied for a variety of medical applications including their use as neuroprotective agents, HIV-1 protease inhibitors, bone-disorder therapy agents and X-ray contrast agents. The water soluble fullerenes find many advantages compared to other known DNA condensing agents: (i) fullerene is known to bind DNA through groove binding and the cationic fullerenes have an increased efficiency of DNA condensation due to the combined electrostatic, groove binding and $\pi-\pi$ interactions; (ii) the high electron density of fullerene will be very helpful for the morphological studies of DNA/fullerene hybrids through HR-TEM analysis in the absence of staining agents; (iii) due to the semiconducting nature of fullerenes, the resultant nanostructures will have conductivity properties which might find applications in molecular electronics; and (iv) the high triplet quantum yield of fullerenes can be used for photo-induced DNA damage studies. 
Amit Sharma asked Richard Cogdell: What applications can we learn from your work, e.g. in the field of bioenergy?

Richard Cogdell responded: Photosynthesis provides a blueprint of how to use solar energy to make fuels. When we understand the biological process at a deep mechanistic level the hope is we can use that information to make more efficient and robust systems for doing the same thing. I should say though that I do not believe that we should try to use the biological system directly as it is too fragile and has not evolved to efficiently make fuel for mankind.

Padmaja P. Mishra addressed Joshy Joseph: Fullerenes are known to produce singlet oxygen, which does damage in the DNA. Do you see any effects of singlet oxygen induced damage on your DNA during the interaction?

Joshy Joseph answered: Fullerene derivatives are known to produce singlet oxygen and hence are used as DNA photo-cleaving agents. In the current paper, we haven't done any photo-induced DNA damage studies.

Ilme Schlichting queried: Have you thought about using metal-doped fulllerenes?

Joshy Joseph replied: We haven't tried metal-doped fullerenes for the current studies.

Ilme Schlichting then asked Richard Cogdell: What is known about the light dependent transcription regulation?

Richard Cogdell answered: The regulation of all of these complexes is at the level of transcription. There is an all or nothing switch controlled by an oxygen sensor kinase and its regulator transcription factor. This is the RegA/RegB system. Then when the oxygen tension is low enough the extent of production of the LH complexes is controlled by both light intensity, by the AppA sensor kinase and its regulator transcription factor PpsR, and by light quality using a set of bacteriophytochromes. The AppA protein, which you determined an X-ray crystal structure for, is well characterised. Very little work has been done on the phytochrome regulation in this system.

Mike Jones said: In one case you see a carbon source dependent change in pigmentation. Do you know the biochemical basis for that?

Richard Cogdell responded: This effect was noted a long time ago by Karin Schmidt but it has never been really investigated. So the answer is we don't know.

Bern Kohler commented: In natural environments, low intensity light generally has a different spectral distribution than high intensity light. Have you looked into how spectral and intensity changes in illumination influence adaptation?

Richard Cogdell responded: In our experiments we make sure that the light intensity changes do not change the spectral composition of the incident light. 
These bacteria do have bacteriophytochromes that can detect changes in the spectral composition but we have not studied that.

Mike Jones asked: You say there are 12 copies of the LH2 structural genes. How different are the protein sequences? Are all of them expressed? How much do we know about these multiple copies?

Richard Cogdell responded: Yes in Rhodopseudomonas acidophila there is this really large puc gene family. The protein sequences have subtle differences, but are all rather homologous. We have not been brave enough to get to grips with this level of complexity. Rather we have tackled the case of Rhodopseudomonas palustris where the puc gene family only has 5 members. In this case we have a full set of all the different puc gene deletions and will soon be able to answer your questions for that species.

Rienk van Grondelle remarked: I understand that these bacteria have amazing adaptability in terms of light harvesting capability. Why have plants lost this?

Richard Cogdell replied: You can never be sure that any answer to this type of question is actually correct. However I suspect that it is because plants typically are nearer the sun and too little light is often not a problem. For many plants, especially those here in India, quickly become light saturated soon after sunrise. Their problem is how to safely get rid of the excess light. They have developed multiple mechanisms to do this.

Amit Sharma said: Nanometals have been shown to improve photosynthetic efficiency in plants. Do you have any thoughts about the mechanism for this?

Richard Cogdell answered: I am sorry but I don't know what this refers to. Plasmonic effects can enhance light harvesting but I am not aware of any data to show this makes plants more efficient.

Ilme Schlichting opened a general discussion of the Concluding remarks lecture by Rienk van Grondelle: Just a comment. I fully agree with what you said. It would be very useful to calculate vibrational spectra using the proposed models and to compare whether they fit to the experimental spectra. However, this may not be so straight-forward in practice, as vibrational spectra code for a lot of details, such as for instance the chromophore-protein interactions. Accurate description of these interactions by computational models is still challenging, but the frequencies, as we know, are sensitive to these interactions. On the other hand, if one considers a (real) many-atom system there are many vibrational modes to consider, some of which have similar frequencies and some of which correspond to the chromophore and protein vibrations mixed. It is often unclear how to assign the computed frequencies to the experimental ones for such a complex system without additional experiments and computations, like for instance site-directed isotope labeling. Experimentally, site-specific isotope labeling is rather demanding...

Roberto Improta remarked: I strongly agree we should investigate the role of solvent molecules in excited dynamics with a lot of attention. We already have 
computational evidence of a strong coupling between the vibrational modes of the solute and those of the first solvation shell, which significantly modulate the spectral line shape. Vibronic coupling with solvent molecules rules a fundamental process as vibrational coupling and should modulate all the different dynamical features, including coherence, of excited state dynamics, especially for charge transfer states. The problems we face when investigating these kind of phenomena in systems as dinucleotides are connected to the existence of many different minima, and many key vibronic parameters exhibit a strong dependence on the geometry considered, making the treatment of the interplay between fast and slow degrees of freedom critical.

Bern Kohler addressed Rienk van Grondelle: In your remarks, you made a compelling case for the role that quantum coherence plays in promoting efficient charge separation in natural light harvesting complexes, but this has also been the subject of vigorous debates. Would you like to comment on the controversies for those outside the field?

Rienk van Grondelle replied: We/I have tried to quantitatively explain our observations on photosynthetic systems using correct physical models. ${ }^{1}$ Such models typically include the coupling between electronic states giving rise to coherences. The environment was included as a classical bath. Since 2014, when we observed the long-lived coherences in photosynthetic reaction centers we have started to include specific vibrations into the quantum model for the electronic states. This implies building a set of quantized vibrational states on each of the electronic states. This leads to a reasonable but not perfect description of the observations. The people who criticize us typically do not do this. They have a very simple, according to me too simple, explanation of the observed spectroscopy, they never show a quantitative fit of their observations using a physical model of photosynthetic energy transfer and charge separation, which I find unsatisfactory.

1 V. I. Novoderezhkin, E. Romero and R. van Grondelle, How exciton-vibrational coherences control charge separation in the photosystem II reaction center, Phys. Chem. Chem. Phys., 2015, 17, 30828-30841.

Dimitra Markovitsi remarked: Fluorescence anisotropy, fluorescence decays and fluorescence spectra, recorded from the fs to the ns time-scales, for both genomic DNA and model oligonucleotides, have brought valuable information on: ultrafast energy transfer involving exciton states, ${ }^{1-3}$ low energy (UVA) mixed $\pi \pi^{*} /$ charge transfer states, ${ }^{4-6}$ and High energy Emitting Long-lived Mixed (HELM) states. ${ }^{7,8}$ It was shown that the collective behaviour of the bases in respect to these effects increases with the rigidity of the system which is affected by the duplex/G-quadruplex size and the ionic strength of the buffered solution. Gquadruplexes exhibit more pronounced collective effects compared to duplexes. ${ }^{9}$

1 D. Markovitsi, D. Onidas, T. Gustavsson, F. Talbot and E. Lazzarotto, J. Am. Chem. Soc., 2005, 127, 17130-17131.

2 D. Markovitsi, T. Gustavsson and I. Vayá, J. Phys. Chem. Lett., 2010, 1, 3271-3276.

3 I. Vayá, T. Gustavsson, T. Douki, Y. Berlin and D. Markovitsi, J. Am. Chem. Soc., 2012, 134, 11366-11368.

4 A. Banyasz, I. Vayá, P. Changenet-Barret, T. Gustavsson, T. Douki and D. Markovitsi, J. Am. Chem. Soc., 2011, 133, 5163-5165. 
5 A. Banyasz, T. Douki, R. Improta, T. Gustavsson, D. Onidas, I. Vayá, M. Perron and D. Markovitsi, J. Am. Chem. Soc., 2012, 134, 14834-14845.

6 A. Banyasz, T. Gustavsson, D. Onidas, P. Changenet-Barret, D. Markovitsi and R. Improta, Chem.-Eur. J., 2013, 19, 3762-3774.

7 M. Huix-Rotllant, J. Brazard, R. Improta, I. Burghardt and D. Markovitsi, J. Phys. Chem. Lett., 2015, 6, 2247-2251.

8 I. Vayá, J. Brazard, M. Huix-Rotllant, A. Thazhathveetil, F. Lewis, T. Gustavsson, I. Burghardt, R. Improta and D. Markovitsi, Chem.-Eur. J., 2016, 22, 4904-4914.

9 P. Changenet-Barret, Y. Hua and D. Markovitsi, Top. Curr. Chem., 2015, 356, 183-202.

Stefan Haacke commented: I would be careful with the phrasing "quantum or optical coherence" because it leads to the misunderstanding that for vibronic coherences to occur one would need a coherent light source for their excitation. Vibronic coherence, i.e. vibrations with a defined phase relation, can exist in each single molecule that absorbs a photon. It is just that we use these coherent short pulse lasers, since we work on molecular ensembles and have to synchronise these to make the coherences among different molecules visible. I understand that you talk about superposition of quantum states, a coherent superposition, of the vibronic states in adjacent molecules in a light harvesting aggregate, and how this coherence survives energy relaxation, correct? Is the idea of "quantum coherence improves reaction efficiency" then simply due to the quantum entanglement of the vibronic wave functions of the initial and final states?

Rienk van Grondelle answered: I use "quantum coherence" on purpose because what we see is a quantum effect, this does not exist in classical physics. The major origin for speeding up the transition rate is 'delocalization' which is a true quantum effect. Because the frequency that couples the two (initial and final) states is very prominent in the spectral density the delocalization is also coupled to fast relaxation (in our model). We are working on a more detailed paper together with Pavel Maly, Doran Bennett and Alan Aspuru-Guzik.

Manas Sarangi asked: How does nature take care of the coherence and decoherence in photosynthetic systems?

Rienk van Grondelle responded: It is not a matter of 'taking care'. The coherences occur due to the strong coupling between the pigments, which with pigment-pigment distances of $1 \mathrm{~nm}$ are unavoidable and large. In photosynthetic antennae excitations are typically delocalized over several pigments and they do not decohere. In addition, different electronic states in the antenna or in the reaction center are resonantly coupled to some vibrations, typically 4 or 5 , and as a consequence these electronic states are further delocalized. This delocalization will indeed decohere, although it is not precisely known how and how fast. This is a subject of intense investigation.

Arijit De said: I would like to add a comment following the last question about whether the noise, apart from (quantum) coherence, influences the energy transfer. Excitation delocalisation spanning over spatially separated chromophores leading to excitonic states is somewhat an obvious outcome of the close proximity of chromophores within an aggregate. To describe the robustness of such energy delocalisation, the "correlated fluctuation" model was proposed by Shaul Mukamel and co-workers which assumes the energy levels of two 
chromophores always remain close. So, we must always talk about exciton energies of dimers or oligomers and not site energies of monomers.

What is a more important problem to look at is how energy transfers between these excitonic states, how a coherent superposition between the excitonic states leads to efficient energy transfer and what is the role of environment (bath) induced fluctuations. In fact, there has been a great body of work, mostly from Martin Plenio's group, trying to address the second question. It has been argued, theoretically, that fluctuations are important to transport energy spatially. You may have a look at this fantastic paper ${ }^{1}$ for more details.

1 F. Caruso, A. W. Chin, A. Datta, S. F. Huelga and M. B. Plenio, Highly efficient energy excitation transfer in light-harvesting complexes: The fundamental role of noise-assisted transport, J. Chem. Phys., 2009, 131, 105106.

\section{Conflicts of interest}

There are no conflicts to declare. 